\title{
BLUE: A Bipedal Robot with Variable Stiffness and Damping
}

\author{
Alexander Enoch*, Andrius Sutas*, Shin'ichiro Nakaoka $\dagger^{*}$ and Sethu Vijayakumar* \\ *Institute of Perception, Action and Behaviour, School of Informatics, University of Edinburgh, United Kingdom \\ $\dagger$ Intelligent Systems Research Institute, National Institute of Advanced Industrial Science and Technology (AIST), Japan \\ E-mail: [a.m.enoch@sms., a.sutas@sms., sethu.vijayakumar@]ed.ac.uk, s.nakaoka@aist.go.jp
}

\begin{abstract}
Exploiting variable impedance for dynamic tasks such as walking is both challenging and topical - research progress in this area impacts not only autonomous, bipedal mobility but also prosthetics and exoskeletons. In this work, we present the design, construction and preliminary testing of a planar bipedal robot with joints capable of physically varying both their stiffness and damping independently - the first of its kind. A wide variety of candidate variable stiffness and damping actuator designs are investigated. Informed by human biophysics and locomotion studies, we design an appropriate (heterogenous) impedance modulation mechanism that fits the necessary torque and stiffness range and rate requirements at each joint while ensuring the right form factor. In addition to hip, knee and ankle, the constructed robot is also equipped with a three part compliant foot modelled on human morphology. We describe in detail the hardware construction and the communication and control interfaces. We also present a full physics based dynamic simulation which matches the hardware closely. Finally, we test impedance modulation response characteristics and a basic walking gait realised through a simple movement controller, both in simulation and on the real hardware.
\end{abstract}

\section{INTRODUCTION}

A key motivator for the development of variable impedance actuators is to create robots which are inherently more robust to disturbances, more energy efficient, or more human-like. A problem which combines all three of these is bipedal locomotion, and it is that problem that we start to tackle in this work, by producing a bipedal robot capable of varying both the stiffness and damping of its joints independently. Human mobility is dominated by walking: our actuation is provided by complex antagonistic arrangements of muscle tendon complexes, each of which exhibits a nonlinear compliant behaviour [1], i.e., the compliance is built into our dynamics. By co-contracting pairs of muscles, we can alter the stiffness of a joint, tensing up when appropriate to deal with the environmental uncertainities and task demands. Such co-contraction also changes the damping of the joint [2]. We deal with walking as a dynamic periodic process, a controlled fall that is typically modelled as a bouncing gait [3].

Robotics has yet to match the ease, energy efficiency, and robustness of human walking. Traditional bipedal robots with rigid joints (e.g. [4], [5]) are energetically inefficient, and not inherently robust to disturbances in terrain. Passive dynamic walkers (e.g. [6]) are incredibly energy efficient, but cannot perform any tasks other than walking at the speed they

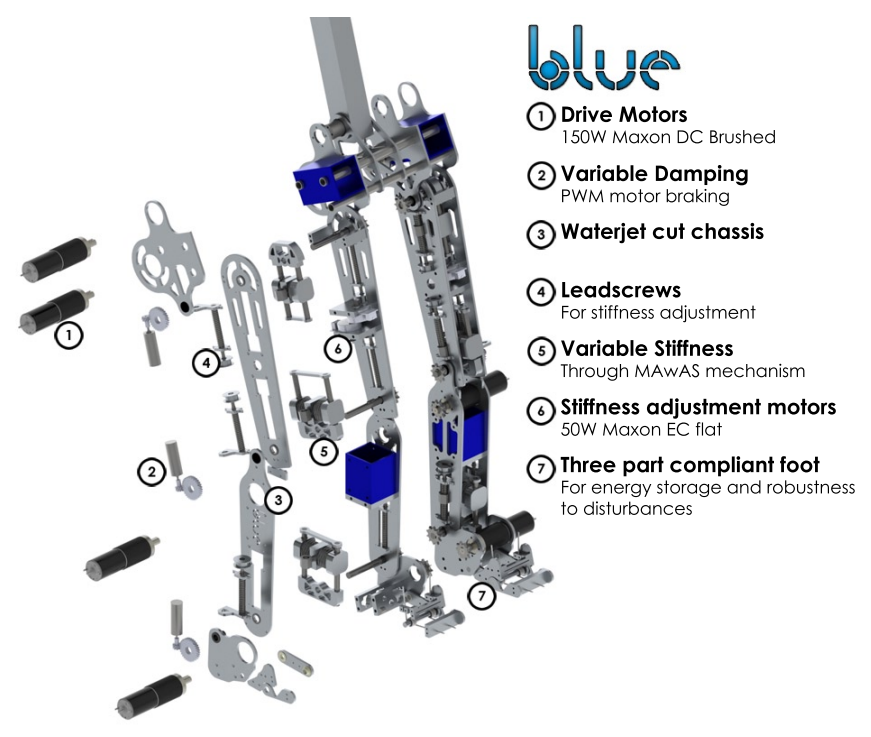

Fig. 1. The planar biped BLUE

were designed for. Torque controlled bipedal robots allow simulation of compliance [7] [8], but without any of the physical advantages that passive compliance provides.

Very few bipedal robots capable of varying joint impedance have been designed. Some have antagonistic non-linear springs [9] or pneumatic muscles [10]; however, these have tended to be slow and not capable of exhibiting dynamic effects. A more agile, antagonistic pneumatic robot is described in [11], but compliance control of its joints is still fairly primitive. Veronica [12] (see also [13], [14]), built around MACCEPA variable stiffness joints, is only capable of achieving relatively low joint stiffness. MABEL [15], a planar biped, uses unidirectional leaf springs to give bounce to its legs, and has used these to store energy during locomotion. However, it is not capable of varying the stiffness of its individual joints and does not have ankle joints or feet.

We therefore aim to develop a bipedal robotic platform which combines the functional flexibility of traditional rigid joint robots with the energy efficiency and robustness to disturbances of passive dynamic walkers. The brief includes a robot that should have fine control over its stiffness and damping, hence, providing a unique platform for research into the effects of physically varying these parameters during 


\begin{tabular}{|cl|c|}
\hline & & Power $\left(\mathbf{W ~ k g}^{-1}\right)$ \\
\hline \multirow{2}{*}{ Summed } & Postive & $0.72 \pm 0.13$ \\
& Negative & $0.37 \pm 0.06$ \\
\hline \multirow{2}{*}{ Hip } & Positive & $0.28 \pm 007$ \\
& Negative & $0.03 \pm 0.03$ \\
\hline \multirow{2}{*}{ Knee } & Positive & $0.12 \pm 0.06$ \\
& Negative & $0.20 \pm 0.06$ \\
\hline \multirow{2}{*}{ Ankle } & Positive & $0.32 \pm 0.08$ \\
& Negative & $0.14 \pm 0.04$ \\
\hline
\end{tabular}

TABLE I

AVERAGE MECHANICAL POWER OVER FULL GAIT CYCLE IN HUMAN WALKING. FROM UMBERGER 2007 [16]

walking and other movements.

By looking at human locomotion studies (e.g. [16]), we see that there is a significant amount of "negative work" done during the gait cycle, that is to say, work which is done on the body from external forces. Table I shows a summary of joint power over a gait cycle at a self selected preferred walking speed. Note that, especially in the knee and ankle, there is a significant amount of negative work done, and we try to design a robot which is capable of storing some of this in order to reduce the amount of positive work required from the motors. In the knee we see that damping would be especially useful for dissipating energy without requiring work from the drive motors.

In order to create a robot with interesting dynamic effects, but one which is not so bulky as to be difficult to manoeuvre in the lab, we define the hip rotation height to be $700 \mathrm{~mm}$ and the target weight to be $25 \mathrm{~kg}$. This makes the robot around $\frac{3}{4}$ of the size of the average American solider [17]. All of the robot's links are scaled from this anthropometric data.

In this paper, we introduce the planar bipedal robot BLUE (Bipedal Locomotion at the University of Edinburgh). We first study the available mechanisms for variable stiffness and variable damping, and select those which seem best suited to actuating a large dynamic walker. The robot itself is then designed around these mechanisms, with powered hip, knee and ankle joints, and a three part compliant foot. We show data from simulations of the platform, and finally show experimental validation of the designed platform and some basic initial movement controllers.

\section{Choosing Mechanisms for Variable Stiffness AND DAMPING}

Joints with variable stiffness or damping are far more complex than a traditional rigid robot joint, and the choice of these mechanisms will have a large bearing on the design of the robot as a whole. Therefore as the starting point for our design we look at the field of variable impedance, to see which types of mechanisms would be most suitable for the task at hand. There have been a great many designs published recently for variable stiffness mechanisms. Fewer have been published for variable damping.

\section{A. Variable Stiffness Mechanisms}

Variable stiffness designs can be broken down into three major categories: antagonistic, series pretension, and tunable springs.
1) Antagonistic: Antagonistic designs typically have two actuators pulling through springs in series, and alter stiffness by co-contracting these springs. It can easily be shown that for co-contraction to change stiffness, non-linear springs must be used, and so most designs for antagonistic variable stiffness focus on creating a suitable non-linear spring (e.g. [18] [11] [19]) or arranging linear springs in such a way as to create non-linearity [20].

Antagonistic designs increase stiffness by co-contracting, this uses a significant amount of energy and implies that some of the energy storage potential of the springs is wasted. Keeping such a joint stiff also involves expending energy, unless the actuators used are non-backdriveable. Furthermore, two powerful actuators are required, unless the compliant elements are bidirectional, or a more complex design is used [21] [22].

2) Series Pretension: Series pretension devices have a spring in series with the actuator, with a design such that the spring can be pre-stressed in order to increase joint stiffness [12] [23] [14]. These mechanisms also share the problem that changing stiffness wastes energy and means that the full energy storage potential of the actuator is not available at all stiffness settings.

3) Tunable Springs: Tunable spring designs rely on changing the geometry of a compliant element in series with an actuator in order to adjust the stiffness (e.g. [24] [25] [26]). Typically these mechanisms may have a smaller compliant range than other designs, but require little energy to change stiffness.

4) The MAwAS Variable Stiffness Mechanism: To get an idea of design requirements for locomotion, we look at typical walking data from human studies, which is normalised by leg length and body mass. We can see that we will need to generate output torques in the region of $30 \mathrm{Nm}$, and whilst the exact amount of compliant deflection required will depend on control strategy, it is generally desirable to have this maximised (certainly larger than $20^{\circ}$ ). We also look at the negative work done on the joints to set a criteria for the minimum amount of energy storage required - for the ankle, this would amount to roughly $3.5 \mathrm{~J}$.

Considering these criteria, as well as the merits and demerits of the various types of variable stiffness mechanisms, we opted to utilise a modified version of the AwAS actuator [24], which we term the Modified AwAS (MAwAS). Our modifications have resulted in increased compliant range and also prevents uneven spring compression. We also use wave springs in order to make the design more compact.

Key advantages of this variable stiffness mechanism are:

- Ability to deliver torques $>30 \mathrm{Nm}$.

- Energy storage $>3.5 \mathrm{~J}$

- Reach low stiffness (to make use of passive dynamics)

- Good range of compliances

- Minimal energy required to change stiffness

- Holding joint at high stiffness requires no energy

- Simple to manufacture

- Energy storage independent of stiffness setting 


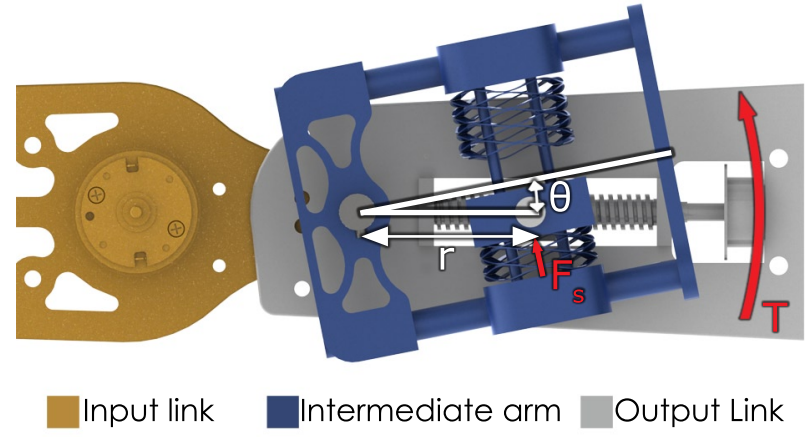

Fig. 2. Schematic of the variable stiffness transmission. The motor attached to the input link drives the intermediate arm which, through a pair of compression springs, delivers torque to the output link.

- Stiffness ranges easily changed by swapping springs

- Becomes a rigid joint when compliant range exceeded, without any damage to springs

5) MAwAS joint dynamics: A schematic of the variable stiffness design is shown in Fig. 2. The joint drive motor on the input link moves an intermediate arm, shown in diagram in blue. This is connected via two compression springs to the output link, shown in grey. A second, smaller, motor is used to adjust the distance of the springs from the axis of rotation $(r)$ by driving leadscrews. As $r$ increases, the amount of compression of the relevant spring for a given deflection from equilibrium $(\theta)$ increases, and thus so does the effective joint stiffness.

Calculating the torque and stiffness functions of the transmission as a function of $r$ and $\theta$ is quite straightforward. The springs used are compression only, and thus, when the transmission is out of equilibrium by angle $\theta$ the compression of the spring, $x$, is given by $x=r \sin \theta$. The spring force $F_{s}$ can then be computed as

$$
F_{s}=r K_{s} \sin \theta
$$

where $K_{s}$ is the spring constant. The torque acting on the output link can thus be calculated by taking the component of the spring force which acts perpendicular to the output arm:

$$
T=\frac{r^{2} K_{s}}{2} \sin (2 \theta)
$$

We define the stiffness to be the derivative of the torque function with respect to $\theta$, and this is given by:

$$
K=\frac{d T}{d \theta}=r^{2} K_{s} \cos (2 \theta) .
$$

When the maximum compression of the spring is reached, the transmission hits a hard stop, and effectively becomes a rigid joint. The maximum deflection required to reach this rigid limit is given by:

$$
\theta_{\max }=\sin ^{-1}\left(\frac{l_{\text {comp }}}{r}\right)
$$

where $l_{\text {comp }}$ is the maximum compression of the spring. With the size and target weight of the robot known, we look
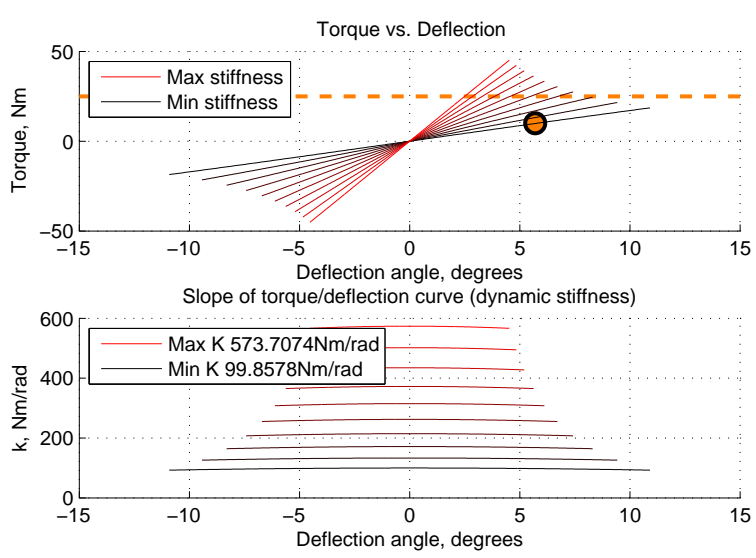

Fig. 3. Torque and stiffness curves for the variable stiffness mechanism in the hips. Predicted required torque/deflection point for walking is shown, and maximum predicted torque for walking shown as dotted line.
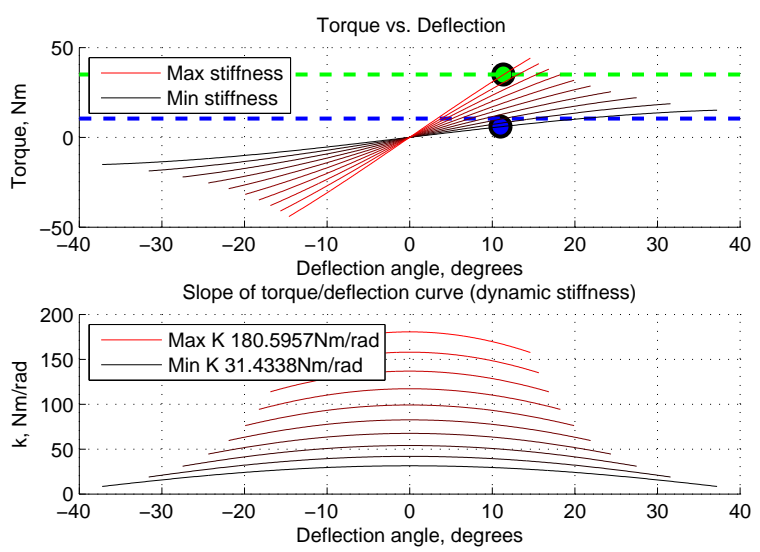

Fig. 4. Torque and stiffness curves for the variable stiffness mechanism in the knees and ankles. Predicted required compliance/torque point is shown in blue for the knee, green for the ankle

at studies of human walking kinetics (e.g. [16]) in order to compare likely required torques with compliant deflections - this aids in the selection of springs for the joints. It should be noted that it is a relatively trivial matter to change the springs in the variable stiffness mechanism in order to change the overall stiffness/deflection characteristics of the joint. For the hip joints, we use springs with $K_{s}=94540 \mathrm{~N} / \mathrm{m}, l_{\text {comp }}=$ $6.15 \mathrm{~mm}$. For the knees and ankles we use springs with $K_{s}=$ $29760 \mathrm{~N} / \mathrm{m}, l_{\text {comp }}=19.66 \mathrm{~mm}$

Fig. 3 shows the torque and stiffness curves for the variable stiffness mechanism in both of the hips of the robot. Similarly, Fig. 4 shows these for the knees and ankles. Each graph also shows the maximum expected joint torque for walking as a dashed line, and uses the required energy storage and torque during an instant in the walking cycle to illustrate a point which will meet these criteria. It is necessary to choose springs which roughly meet the energy storage criteria, but also can be stiff enough to provide high torque for movements not just limited to walking.

6) Energy Storage in the MAwAS: The energy stored in the compliant transmission can easily be shown to be $U_{e}=$ 


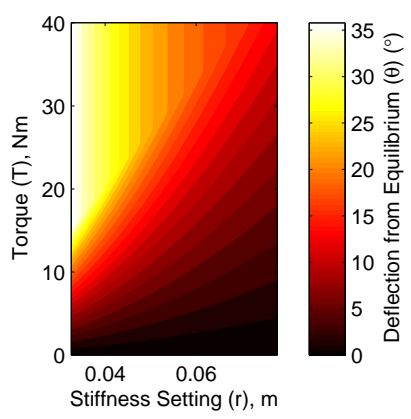

(a) Resultant Deflection

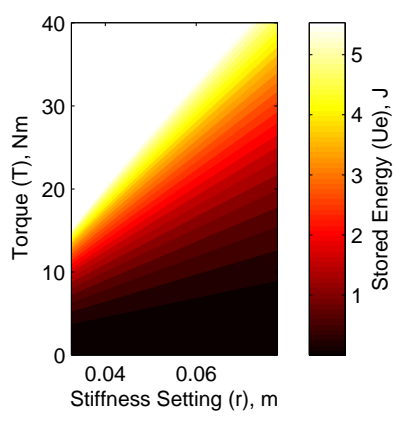

(b) Stored Energy
Fig. 5. Stored energy in the MAwAS for different stiffness settings and torques. (a) The deflection from equilibrium caused by an applied torque, for various stiffness settings. (b) The energy stored in the springs of the variable stiffness joint for the same applied torque and stiffness settings.

$\frac{1}{2} K_{s} r^{2} \sin ^{2} \theta$. The deflection from equilibrium is shown as a function of output torque and stiffness setting in Fig. 5(a), and the resulting energy stored in Fig. 5(b). These graphs are for the springs used in the knee and ankle joints.

7) Energy Cost of Changing Stiffness: A key concern in the choice of a mechanism for variable stiffness is the energy cost of changing stiffness. Since the MAwAS mechanism does not rely on pretension in order to change compliance, the energy used to change the stiffness setting is quite small. We consider the energy required to increase stiffness when the joint is out of equilibrium, keeping the energy stored in the system the same. In order to move the spring carriage and increase stiffness the component of spring force acting down the output link must be considered, this is given by $F_{p}=F_{s} \sin \theta$. Using eq. (1) it follows that:

$$
F_{p}=\frac{F_{s}^{2}}{r K_{s}}
$$

The energy used working against this force to change stiffness can therefore be calculated by a simple integration:

$$
\begin{aligned}
E & =\int_{r_{1}}^{r_{2}} F_{p} d r \\
& =\frac{F_{s}^{2}}{K_{s}}\left(\ln \left(r_{2}\right)-\ln \left(r_{1}\right)\right)
\end{aligned}
$$

Using realistic numbers from our implementation, the total energy required to move from $r_{1}=0.0325$ to $r_{2}=0.078$ against a spring force of $F_{s}=300 \mathrm{~N}$, with a spring constant $K_{s}=29760 \mathrm{~N} / \mathrm{m}$ would use around $2.6 \mathrm{~J}$ of energy. With lead screw transmission efficiency of $44 \%$ the energy used by the stiffness adjustment actuator should be around $6 \mathrm{~J}$. This is a very simplified calculation which does not include additional frictional forces, and assumes that the spring carriage itself is massless. Nevertheless, the power required to adjust the stiffness is very small compared to the power required to drive the joints.

\section{B. Variable Damping Mechanisms}

There have not been as many designs published for mechanisms to achieve variable damping. Some utilise Magneto-

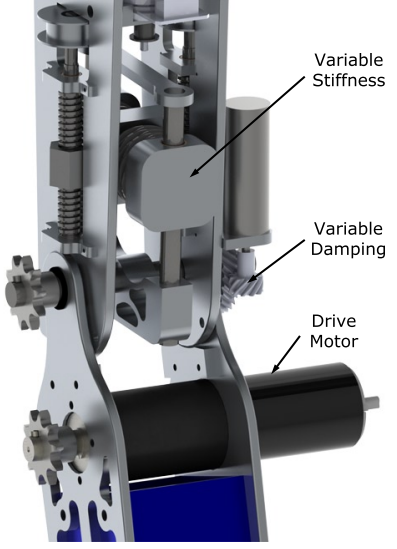

(a) Variable Impedance Joint

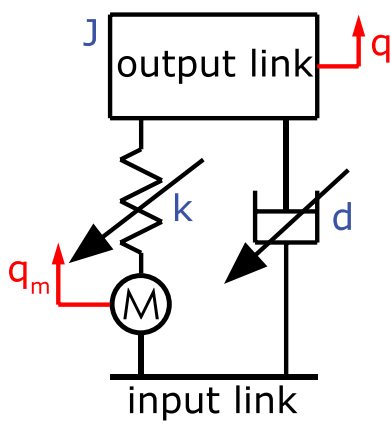

(b) Joint Dynamics
Fig. 6. (a) Implementation of variable stiffness and variable damping. Helical gearing is used to connect the damping motor in parallel with the variable stiffness drive. (b)Translational equivalent of the joint dynamics. Each joint has variable stiffness and damping. The drive motor adjusts the equilibrium position of the joint.

Rheological fluid modulated with electromagnetic fields [27], variable frictional damping [26] [28], changing the channel size in a hydraulic damper [18], or PWM variation of motor braking. [29]

Since the majority of these methods require the expenditure of energy in order to apply damping [27] [26] [28], or at the least to adjust it [18], we select the method which uses very little energy to apply and adjust damping - motor braking. This method involves connecting another motor in parallel with the series compliant transmission, and shorting the terminals of the motor in order to brake it and apply damping torque. By modulating this with PWM we can continuously vary the achieved damping, with the energy absorbed being dissipated as heat in the motor windings. By utilizing a more sophisticated circuitry, this method could in fact be used to generate energy, in the same way as regenerative braking in modern vehicles - perhaps eventually even transferring this energy between joints to achieve the same effect as bi-articulate joints in the human legs.

The calculation of the exact applied damping is somewhat complex (see [29]), but the maximum achievable damping coefficient can be seen to be

$$
d=\frac{n^{2} \kappa_{\tau} \kappa_{\dot{q}}}{R_{e}}
$$

where $n: 1$ is the gear ratio, $\kappa_{\tau}$ and $\kappa_{\dot{q}}$ are the motor torque and speed constants respectively, and $R_{e}$ is the equivalent resistance of the motor. The damping motor is connected in parallel with the variable stiffness drive, as shown in Fig. 6(a).

\section{JOINT DYNAMICS}

Fig. 6(b) shows a schematic of one of the principal joints of the robot, with variable stiffness and damping. The equation of motion for this can be seen to be:

$$
J \ddot{q}+d \dot{q}+k\left(q-q_{m}\right)=0
$$




\begin{tabular}{|l|l|l|}
\hline Measurement & Human & Robot \\
\hline Hip (Trochanteric) height & 927 & 695.25 \\
\hline Knee height, midpatella & 504 & 378 \\
\hline Foot length & 269 & 201.75 \\
\hline Ball of foot length & 195 & 146.25 \\
\hline
\end{tabular}

TABLE II

SIZING DATA AND CORRESPONDING $\frac{3}{4}$ SCALE SIZES USED IN THE ROBOT. ALL DIMENSIONS IN MM.

Defining $\theta=q-q_{m}$ as the deflection from equilibrium and using eq. (3), this can be rewritten as:

$$
\ddot{\theta}+\frac{d}{J} \dot{\theta}+\frac{r^{2} K_{s}}{J} \cos (2 \theta) \theta=-\ddot{q_{m}}-\frac{d}{J} \dot{q_{m}}
$$

For small oscillations around $\theta=0, \cos (2 \theta) \approx 1$. Therefore, the above dynamics can be simplified as

$$
\ddot{\theta}+2 \zeta \omega_{n} \dot{\theta}+\omega_{n}^{2} \theta=-\ddot{q_{m}}-2 \zeta \omega_{n} \dot{q_{m}}
$$

where $\omega_{n}=\sqrt{\frac{r^{2} K_{s}}{J}}$ is the natural frequency and $\zeta=$ $\frac{d}{2 \sqrt{r^{2} K_{s} J}}$ is the damping ratio. Thus, by changing $r$ and $d$, we can change the natural frequency and damping behaviour of the joint.

In a more general sense, the equation of motion for the entire robot can be seen to be:

$$
M(q) \ddot{q}+C(q, \dot{q}) \dot{q}+g(q)=T(\theta)+D \dot{q}+F_{\text {ext }}(q, \dot{q}, \ddot{q})
$$

where $M$ is the inertia matrix, $C$ represents Coriolis forces, $g$ introduces forces from gravity, $T$ is the torque from the variable stiffness transmission $\left(T_{i}=\frac{r_{i}^{2} K_{s i}}{2} \sin \left(2 \theta_{i}\right)\right), D$ represents the variable damping, and $F_{\text {ext }}$ includes external forces, for e.g., due to ground contact forces.

\section{Design And Construction of BLUE}

BLUE has been built and initial testing completed. So far, the major joints and feet have all been implemented, with variable stiffness functionality, but variable damping has not yet been physically added.

\section{A. Mechanical Design}

BLUE is a planar biped, with powered hip, knee and ankle joints, each of which has variable stiffness and variable damping. There are two completely passive joints in each foot, and a powered torso joint, although this is only position controlled. In this work we present BLUE purely with variable stiffness, variable damping has been designed in but not yet implemented, and we will introduce this in a future paper. Similarly the torso joint and upper body will not feature here. The link lengths of BLUE are scaled from human anthropometric data [17], and are shown in Table II. The target weight of the robot is around $25 \mathrm{~kg}$, and without the torso or damping motors the current weight is around $18 \mathrm{~kg}$. The robot is supported laterally by a rotating boom arm, but is free to move in the sagittal plane.

Mimicking the design and function of the human foot, each of the feet are composed of three parts, with two sprung joints

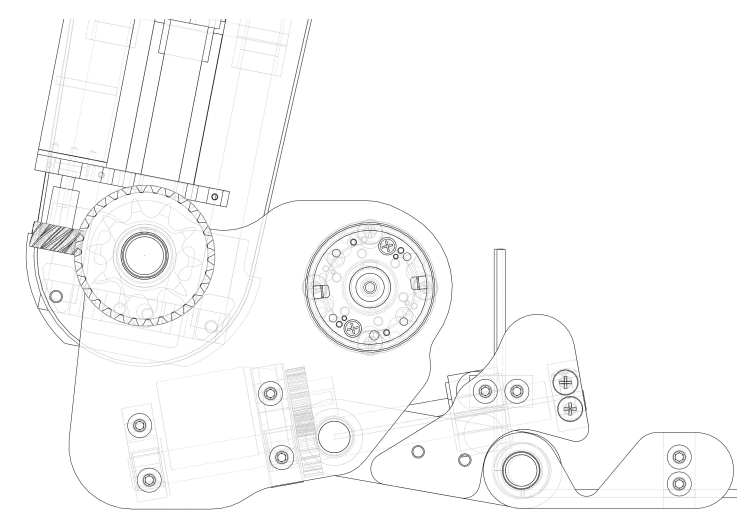

Fig. 7. Foot with compliant arch and toes

creating the longitudinal arch and toes, as shown in Fig. 7. The flexibility of the foot is important as it allows it to mould to the contours of the ground, increasing stability, as well as storing energy in ways a rigid foot could not [30] [31]. A compliant foot also gives a better roll-over shape than a rigid foot, supporting the robot more as it approaches toe-off. The design of the feet also allows for the easy implementation of a windlass mechanism, which stiffens the foot as the toes extend [32].

The main six joints of the robot are all based around the variable stiffness mechanism and motor braking variable damping as detailed before. When selecting drive motors, we looked at the likely torques and velocities which will be required, added a safety factor accounting for gearing efficiency losses, and selected an available motor which best meets the corresponding requirements. All six of the drive motors are $150 \mathrm{~W}$ Maxon RE40 DC motors with planetary gearheads. The final stage of the transmission is a chain drive, and it is a relatively simple matter to adjust the final gearing ratio by changing the ratio of these sprockets. The stiffness adjustment motors are 50W Maxon EC45 flat brushless DC motors, with a simple timing belt drive to the leadscrews, allowing fast adjustment of stiffness. Joint angle requirements are taken from human movement data, with a mechanical stop preventing hyperextension of the knee joints and helping to bear load during the stance phase of walking.

The chassis of the robot is designed to be waterjet cut, in order to reduce the construction time. These parts are shown (not to scale) in Fig. 8(a), and the robot itself is shown in Fig. 8(b). Waterjet cut parts were machined in a standard manual mill to create any three dimensional features required.

\section{B. Sensors and Electronics}

Each variable impedance joint on the robot has two magnetic encoders to give the rotary positions of the intermediate arm and output link. Since we know the spring deflection and torque transfer function, it is also possible to calculate torque in the joints with this data. The drive motor electronics also include current sensing. The position of the spring carriage, and hence the stiffness setting, is measured by a simple linear 


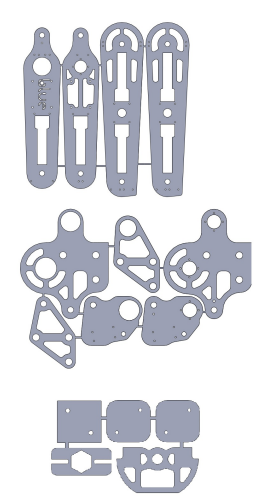

(a)

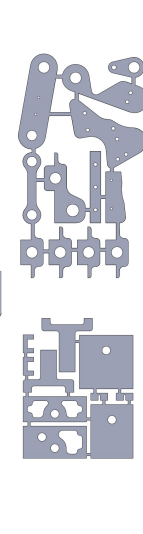

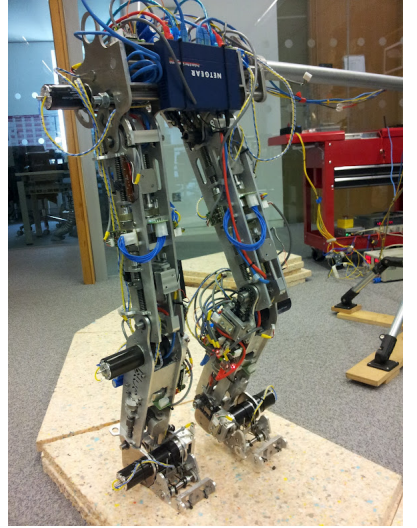

(b)
Fig. 8. (a) The waterjet cut parts from which BLUE is built. (b) The constructed robot.

\begin{tabular}{|c|c|c|}
\hline Sensor & Type & Implementation \\
\hline $\begin{array}{l}\text { Joint angle \& } \\
\text { Intermediate arm } \\
\text { angle }\end{array}$ & Rotary position & $\begin{array}{l}\text { Magnetic encoders } \\
\text { (Austria Microsystems } \\
\text { AS5040-ASSU) }\end{array}$ \\
\hline Stiffness setting & Linear position & $\begin{array}{l}\text { Linear potentiometer (Bourns } \\
\text { PTA6043-2015DP-B103) }\end{array}$ \\
\hline Joint torque & Calculated & Virtual \\
\hline Drive motor current & Analog readout & Built in to ESC \\
\hline $\begin{array}{l}\text { Ground reaction } \\
\text { force }\end{array}$ & $\begin{array}{l}\text { Force magnitude } \\
\text { and distribution }\end{array}$ & $\begin{array}{l}\text { Six Force } \\
\text { Sensitive Resistors } \\
\text { (Interlink FSR402) }\end{array}$ \\
\hline Vestibular & $\begin{array}{l}\text { Inertial } \\
\text { measurement } \\
\text { and tilt }\end{array}$ & $\begin{array}{l}\text { 3-axis accelerometer \& gyros } \\
\text { (ADXL335, LY530AL, } \\
\text { LPR530AL, HMC5843) }\end{array}$ \\
\hline
\end{tabular}

TABLE III

SUMMARY OF THE SENSORS ON BLUE

potentiometer. Six force sensitive resistors on each foot of the robot give information about ground reaction force on each of the three foot segments. Finally a 3 -axis accelerometer and 3-axis gyro mounted on the torso of the robot give inertial measurement and tilt data. A summary of the sensors on the robot is given in table III.

The architecture of the electronics on the robot is shown in Fig. 9. A control board with ATMEL microcontroller (AT91SAM7x256) and Ethernet interface is present on each joint of BLUE. This communicates with a fitPC2 $\mathrm{i}$ embedded computer running Arch Linux with a real time kernel. The fitPC runs the control program, sending target trajectories to the control boards of each joint. These boards interface with motor driver boards for each of the three motors on the joint, running PID loops for control of the drive and stiffness adjustment motors.

The control boards also read any relevant sensors, filter the data, and relay it back to the fitPC. For rotary sensor data from the magnetic encoders, a Synchronous Serial Interface (SSI) is used to obtain a digital readout from the encoder with 10 bit accuracy. This is then filtered through a moving average filter on the control board to give a very steady signal from these sensors. The other sensors - stiffness position, motor current, etc - are read through the ADC. All signals

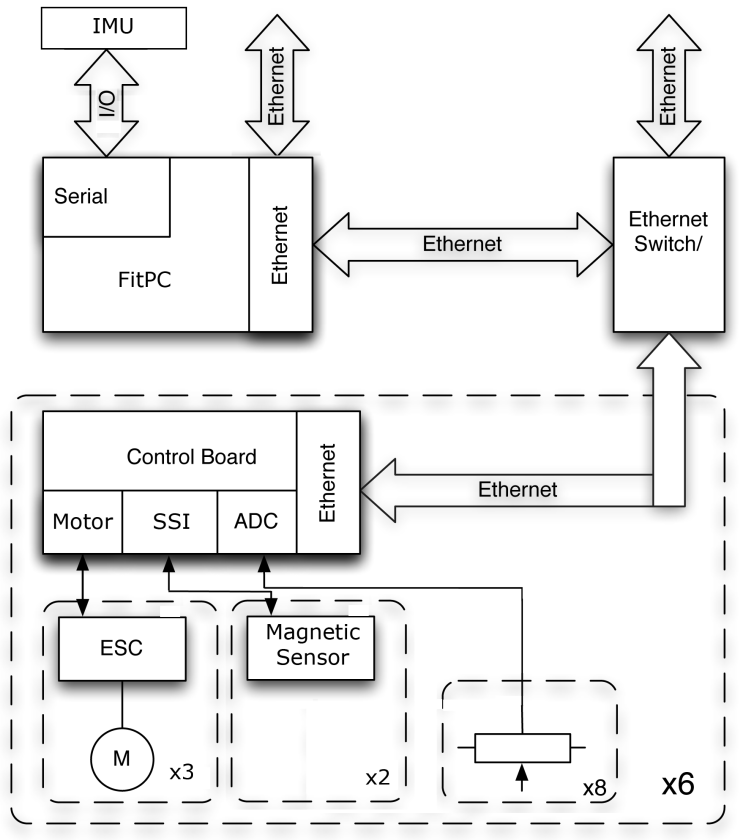

Fig. 9. Schematic of the electronics architecture on board BLUE

are sampled at $1 \mathrm{kHz}$. An on board 8-port Ethernet switch is used to connect together the control boards and fitPC, and a wireless link is used to give high level commands to the fitPC from a remote computer.

\section{EXPERIMENTS}

In addition to building the hardware, we have developed a full physics dynamic simulation using the Choreonoid program [33] to effectively evaluate potential control strategies prior to testing them on the actual hardware. The results of implementing some basic controllers in both simulation and hardware will be discussed here. A video of these experiments is available online.

The CAD model of the robot was ported into Choreonoid with the appropriate mass and inertia properties, and the compliant joints were modelled with the appropriate spring transfer functions. Choreonoid allows for simulation in 2D (meaning the boom arm does not have to be modelled) and can simulate springs and dampers in the joints [34], including non-linear ones. The simulator runs faster than real time on a modern laptop.

Whilst the simulation results can not be expected to exactly match the performance on the hardware, they should allow for the evaluation and testing of potential controllers.

The hardware has been constructed as detailed above, complete with electronics capable of position or torque control, and turning off motors if motor current or joint position limits are breached. This gives a very fast fail-safe system which will prevent the hardware being damaged in the event that a control program does not perform as expected.

It was verified that the joints performed as expected, and particularly that the stiffness adjusters were capable of varying stiffness in a timely manner. Experiments show 


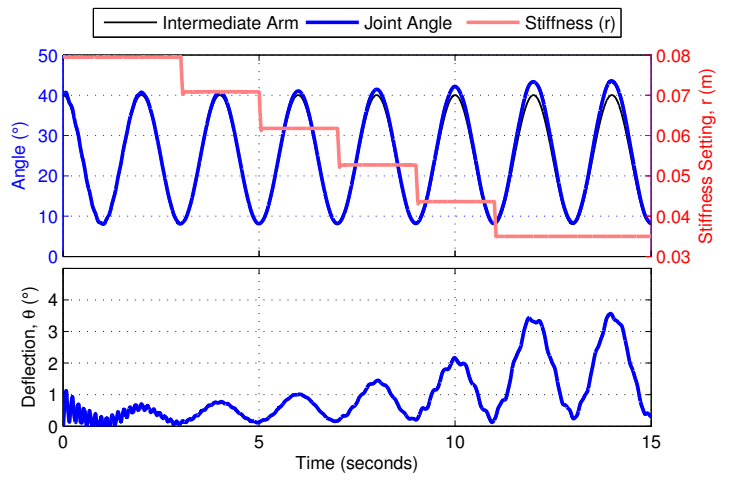

(a) Simulation

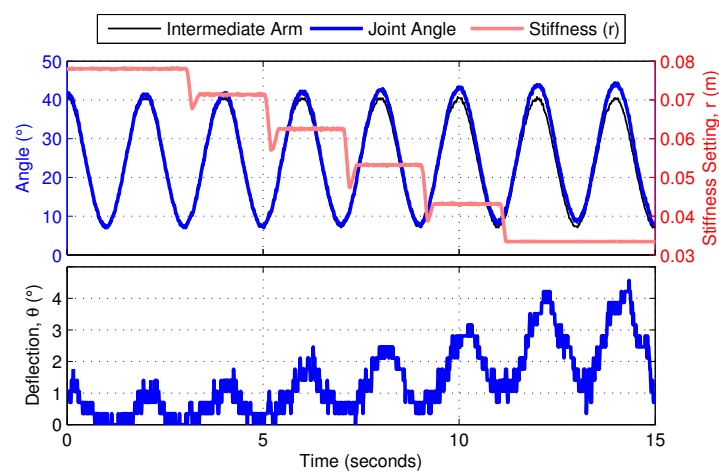

(b) Hardware

Fig. 10. Knee joint during robot squatting under different stiffness settings, both in simulation (a) and on the real hardware (b). Joint stiffness at all joints decreases, and it can be seen that the compliant deflection increases as stiffness decreases. The real hardware exhibits backlash which increases as stiffness decreases, but the same trend can be seen in both graphs. Since the controller gains were set high on the stiffness adjuster, some overshoot can be observed in the stiffness position.

that under no loading, the joint can vary from highest to lowest stiffness and back again within one second. This speed decreases as loading increases, mainly due to additional unmodelled frictional forces with the leadscrews. The physical hardware also experiences backlash on the joints, which is not modelled in the simulation.

The electronics and on-board Ethernet network perform very well, with no detected packet loss. Noise on the angle sensor readings is typically in the region of $0.3^{\circ}$.

\section{A. Squatting}

To provide a basic evaluation of the hardware without the need to worry about issues such as balance, we first conduct experiments with the robot performing continuous squats while the stiffness is changed. This demonstrates that the compliance of the robotic joints does change, and that this has physical results on the behaviour of the robot under a constant control scheme. For these tests, we perform high gain positional control on the intermediate arm, leaving the final link output position compliant through the variable stiffness mechanism.

Fig. 10 shows the commanded joint trajectories and link output positions for one of the knees of the robot, both in simulation and on the actual hardware. Similar graphs are

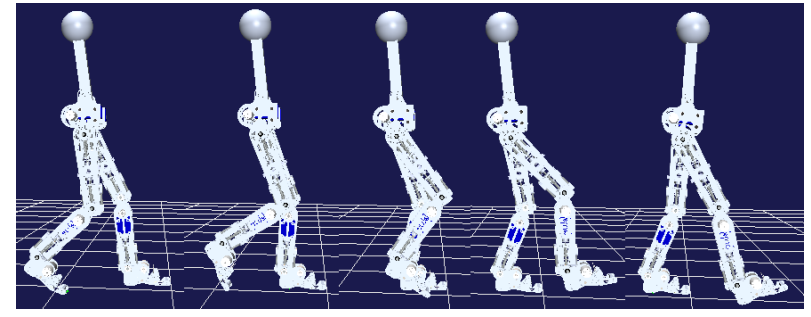

(a) Simulation

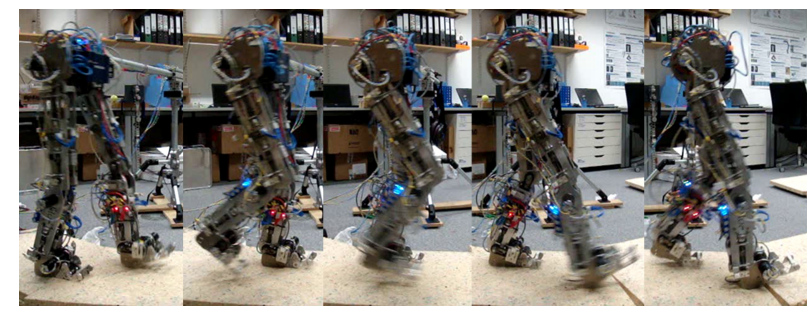

(b) BLUE

Fig. 11. Walking trajectory playback on the robot, both in simulation and on the actual hardware.

observed for the ankles and hips. Both legs are given the same commands, and exhibit the same behaviour. We can observe that the effect of the compliance is noticeable even under these low velocity double-support movements. The hardware follows the same general trend as the simulation, but with some additional deflection due to backlash in the joint. Fig. 10(b) also shows the performance of a stiffness adjuster on the real hardware, and it can be observed that it is capable of rapidly altering the compliance of the joint.

\section{B. Walking}

Since the morphology of the robot is a scaled version of a human, and balance is simplified by the boom arm, we are able to use human walking data from motion capture in order to quickly produce a walking motion. This will not be the most efficient walk possible for the robot, since its mass distribution and joint dynamics are different from a human, but it is a good starting point. First we replay the joint data from human walking with a high stiffness setting, to validate that it does indeed produce stable walking behaviour on the robot. Fig. 11 shows scenes from the walking sequence in simulation and on the physical robot.

When a trajectory was successful on the simulator, it was played back on the hardware. Fig. 12 shows a walking motion on the ankle and knee joints of one leg. In the absence of a torso to aid balance, it was necessary to add a small counterweight to the boom arm, relieving approximately a third of the weight from the robot. The dynamic loading of the joints can be seen in their deflection from equilibrium, as for these experiments only the equilibrium position is being controlled.

\section{CONCLUSION}

Variable impedance technology can be extremely beneficial to bipedal robots, in helping them to be inherently robust to disturbances, and potentially more efficient in their 


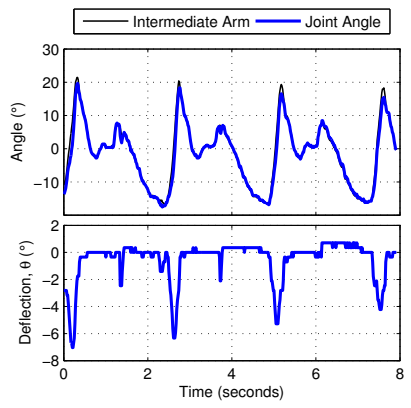

(a) Ankle

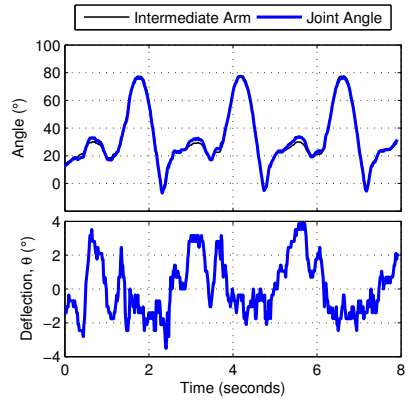

(b) Knee
Fig. 12. Joint trajectories for the ankle and knee of one leg of the physical robot during walking. Stiffness is kept constant at close to the highest setting, and the loading of the joints can be seen through their deflection from equilibrium.

locomotion. We evaluated the wide spectrum of literature of variable impedance actuation in order to select appropriate methods for variable stiffness (tunable springs) and variable damping (motor braking) which seem most suitable for providing the required torque and stiffness required in bipedal locomotion, but which, importantly, can maintain joints at a high stiffness without requiring the expenditure of energy.

We looked at the dynamics of human walking to design a robot which should be capable of mimicking such motion, and have shown both in simulation and on the actual hardware, that walking can be achieved. Furthermore we have shown that the variable stiffness mechanism in BLUE is capable of changing the stiffness of its joints to noticeably affect its dynamics. We have also designed a robust, compact, set of electronics and sensors to control the robot. Future work will concentrate on the performance of the variable damping, and on control strategies for best utilising the variable dynamics of the robot.

\section{REFERENCES}

[1] C. Maganaris and J. Paul, "In vivo human tendon mechanical properties," The Journal of Physiology, vol. 521 (1), p. 307, 1999.

[2] M. F. Eilenberg, H. Geyer, and H. Herr, "Control of a powered anklefoot prosthesis based on a neuromuscular model." IEEE Trans. on Neural Systems and Rehabilitation Engineering, vol. 18(2), pp. 16473, 2010.

[3] H. Geyer, A. Seyfarth, and R. Blickhan, "Compliant leg behaviour explains basic dynamics of walking and running." Proc. Biological sciences / The Royal Society, vol. 273 (1603), pp. 2861-7, 2006.

[4] K. Hirai et al., "The development of Honda humanoid robot," IEEE Int. Conf. on Robotics and Automation, pp. 1321-1326, 1998.

[5] K. Kaneko et al., "Cybernetic human HRP-4C," IEEE-RAS Int. Conf. on Humanoid Robots, pp. 7-14, 2009.

[6] S. Collins et al., "Efficient bipedal robots based on passive-dynamic walkers." Science, vol. 307 (5712), pp. 1082-5, 2005.

[7] S.-h. Hyon and G. Chengtt, "Gravity Compensation and Balancing for Humanoid Robots," in IEEE-RAS Int. Conf. on Humanoid Robots, 2006, pp. 214-221.

[8] C. Ott et al., "Development of a Biped Robot with Torque Controlled Joints," in IEEE-RAS Int. Conf. on Humanoid Robotics, 2010, pp. 167-173.

[9] J. Yamaguchi, D. Nishino, and A. Takanishi, "Realization of dynamic biped walking varying joint stiffness using antagonistic driven joints," IEEE Int. Conf. on Robotics and Automation, pp. 2022-2029, 1998.

[10] B. Vanderborght et al., "Overview of the Lucy Project: Dynamic Stabilization of a Biped Powered by Pneumatic Artificial Muscles," Advanced Robotics, vol. 22 (10), pp. 1027-1051, 2008.
[11] K. Hosoda et al., "Pneumatic-driven jumping robot with anthropomorphic muscular skeleton structure," Autonomous Robots, vol. 28 (3), pp. 307-316, 2009.

[12] R. Van Ham et al., "MACCEPA, the mechanically adjustable compliance and controllable equilibrium position actuator: Design and implementation in a biped robot," Robotics and Autonomous Systems, vol. 55 (10), pp. 761-768, 2007.

[13] Y. Mao et al., "A reinforcement learning based dynamic walking control," in IEEE Int. Conf. on Robotics and Automation, 2007, pp. 3609-3614.

[14] M. Uemura and S. Kawamura, "A new mechanical structure for adjustable stiffness devices with lightweight and small size," in IEEE/RSJ Int. Conf. on Intelligent Robots and Systems, 2010, pp. 2364-2369.

[15] J. Grizzle et al., "MABEL, a new robotic bipedal walker and runner," in American Control Conf., 2009, pp. 2030-2036.

[16] B. R. Umberger and P. E. Martin, "Mechanical power and efficiency of level walking with different stride rates." The Journal of Experimental Biology, vol. 210 (18), pp. 3255-65, 2007.

[17] D. Wagner et al., "Human factors design guide," FAA William J. Hughes Technical Center, 1996.

[18] K. Laurin-Kovitz, J. Colgate, and S. Carnes, "Design of components for programmable passive impedance," IEEE Int. Conf. on Robotics and Automation, pp. 1476-1481, 1991.

[19] G. Tonietti, R. Schiavi, and A. Bicchi, "Design and Control of a Variable Stiffness Actuator for Safe and Fast Physical Human/Robot Interaction," IEEE Int. Conf. on Robotics and Automation, pp. 526$531,2005$.

[20] D. Mitrovic et al., "Exploiting sensorimotor stochasticity for learning control of variable impedance actuators," in IEEE-RAS Int. Conf. on Humanoid Robots, 2010, pp. 536-541.

[21] J. Hurst, J. Chestnutt, and A. Rizzi, "An actuator with physically variable stiffness for highly dynamic legged locomotion," IEEE Int. Conf. on Robotics and Automation, pp. 4662-4667 Vol.5, 2004.

[22] F. Petit et al., "Bidirectional antagonistic variable stiffness actuation: Analysis, design \& implementation," in IEEE Int. Conf. on Robotics and Automation, 2010, pp. 4189-4196.

[23] S. Wolf and G. Hirzinger, "A new variable stiffness design: Matching requirements of the next robot generation," IEEE Int. Conf. on Robotics and Automation, pp. 1741-1746, 2008.

[24] A. Jafari et al. "A Novel Actuator with Adjustable Stiffness (AwAS)," in IEEE/RSJ Int. Conf. on Intelligent Robots and Systems, 2010, pp. 4201-4206.

[25] A. Jafari, N. Tsagarakis, and D. Caldwell, "Awas-ii: A new actuator with adjustable stiffness based on the novel principle of adaptable pivot point and variable lever ratio," in IEEE Int. Conf. on Robotics and Automation, 2011, pp. 4638-4643.

[26] T. Morita and S. Sugano, "Development of an anthropomorphic forcecontrolled manipulator WAM-10," in Int. Conf. on Advanced Robotics, 1997, pp. 701-706.

[27] H. Herr and A. Wilkenfeld, "User-adaptive control of a magnetorheological prosthetic knee," Industrial Robot: An International Journal, vol. 30 (1), pp. 42-55, 2003.

[28] M. Laffranchi et al., "A Variable Physical Damping Actuator (VPDA) for Compliant Robotic Joints," in IEEE Int. Conf. on Robotics and Automation, 2010, pp. 1668-1674.

[29] A. Radulescu et al., "Exploiting variable physical damping in rapid movement tasks," in IEEE/ASME AIM Conf, 2012.

[30] L. Klenerman and B. Wood, The Human Foot: a Companion to Clinical Studies. Springer Verlag, 2005.

[31] A. Gefen, "The in vivo elastic properties of the plantar fascia during the contact phase of walking." Foot \& Ankle International, vol. 24 (3), pp. 238-44, 2003

[32] J. H. Hicks, "The mechanics of the foot. IV. The action of muscles on the foot in standing." Acta Anatomica, vol. 27 (3), pp. 180-92, 1956.

[33] "Choreonoid official site," http://choreonoid.org/.

[34] S. Nakaoka et al., "Constraint-based dynamics simulator for humanoid robots with shock absorbing mechanisms," in IEEE/RSJ Int. Conf. on Intelligent Robots and Systems, 2007, pp. 3641-3647. 